\title{
TOWARDS THE MODELLING OF THE HYDRODYNAMIC FORCES IN COMPOUND OPEN CHANNEL WITH VEGETATED FLOOD PLAIN
}

\author{
by Dan NAOT*, Iehisa NEZU** and Hiroji NAKAGAWA***
}

\begin{abstract}
Steps towards the prediction of the hydrodynamic forces in the compound open channel with vegetated flood plain are described. An algebraic stress turbulence model is offered with options for open surface proximity and for the presence of vegetation. The efficiencies of the production of turbulence energy and dissipation by the vegetation are discussed. Results for the secondary currents and streamwise velocity are shown for dilute vegetation and for dense vegetation at the flood plain.
\end{abstract}

Keywords: open channel, compound, vegetated zones, algebraic stress model, hydrodynamic forces.

\section{INTRODUCTION}

Step towards a numerical study of the hydraulic behavior of compound open channel flow with vegetated zones are here described. These follow both the theoretical study in compound open channel flow of Prinos, Townsend and Tovoularis [7], Krishnappan and Lau [2], Kawahara and Tamai [1], Naot Nezu and Nakagawa $[4,5]$ and others, and the theoretical study in vegetated open channel flow by Shimizu, Tujimoto and Nakagawa [8], Shimizu and Tujimoto [9] and others. To include vegetation its resistance to the flow is estimated and smeared homogeneously turning the vegetated zone into a special class of porous media. Vegetation also produce turbulence which may dominate the intensity, the length scales and the anisotropy of the turbulent fluctuations. Therefore the preliminary steps should refer to a few aspects: Is the mean velocity attenuated equally in all three directions, do secondary currents exist, can the turbulence model cope with wide vegetated domains, can the model describe transition to non vegetated zones smoothly, and others. These are the main topics of the present work.

\section{HYDRODYNAMIC MODEL}

The set of equations solved consists of three groups of equations which together form the hydrodynamic model: Three momentum equations plus the continuity equation governing the mean flow, two transport equations for the energy and dissipation representing the intensity and scale of turbulence and a set of algebraic equations describing the anisotropy of the turbulent stresses. The basic set up is well reported $[4,6]$ and therefore only the features introduced to cope with the presence of vegetation are described. The solution domain and the coordinates are shown in Fig.1.

The resistance of the vegetation to the mean flow is modeled by three body forces added to the momentum equations:

\footnotetext{
* Assoc. Prof., Center for Techological Ed.-Holon, Affiliated to Tel-Aviv Univ., Holon 58102, Israel.

** Assoc. Prof., Dept. of civil Engrg., Div. of Global Envir. Engrg., Kyoto Univ., Kyoto 606, Japan.

*** Prof. Dept. of civil Engrg., Div. of Global Envir. Engrg., Kyoto Univ., Kyoto 606, Japan.
} 
with

$$
F_{x}=C_{x} K U, \quad F_{y}=C_{y} K V \quad \text { and } \quad F_{z}=K W
$$

$$
K=\frac{1}{2} \rho \sqrt{U^{2}+V^{2}+W^{2}}<n><D>C_{D} c(m)
$$

Here, $<n>$ is the averaged vegetation density (Rods per square meter), $\angle \mathrm{D}\rangle$ is the averaged diameter, $C_{D}$ is the drag coefficient corresponding to $\angle \mathrm{D}>$ and $\mathrm{c}(\mathrm{m})$ is a statistical correlation here taken as one. In the present work:

$$
C_{D}=\left(1000 / R_{e}\right)^{0.25}, C_{x}=C_{y}=1 \text { for } R_{e}<<1000
$$

and

$$
\begin{gathered}
C_{D}=\text { minmum of }: 0.976+\left(\frac{R_{e}-2000}{20500}\right)^{2} \text { or } 1.15, C_{x}=1, \\
C_{y}=\left(1000 / R_{e}\right)^{0.2} / C_{D} \quad \text { for } 1000<R_{e}<40000,
\end{gathered}
$$

with $R_{e}=W<D>/ v$ representing the vegetation Reynolds number. The equations for $\mathrm{Cy}$ are based on the assumption that Fy is proportional to the skin friction only with pressure drag disregarded in parallel to the vertical vegetation.

The production of turbulence by the vegetation is modeled by two new production terms introduced into the energy equation:

and the dissipation equation:

$$
\pi_{v k}=\pi_{v} \eta_{k}
$$

$$
\pi_{v \varepsilon}=C_{\varepsilon 1}\left(\pi_{\nu} \frac{\varepsilon}{k}\right) \eta_{\varepsilon}
$$

where $\eta_{k}$ and $\eta_{\varepsilon}$ are the process efficiencies and

$$
\pi_{v}=F_{x} U+F_{y} V+F_{z} W
$$

The implications of the choice of $\eta_{\varepsilon}$ and $\eta_{k}$ will be later discussed.

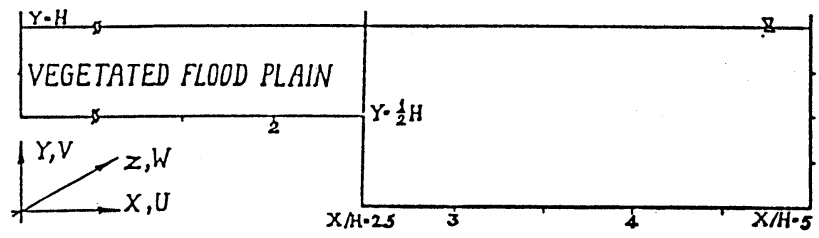

Fig.1. The Compound Open Channel; The Coordinates System

\section{ALGEBRAIC STRESS MODEL}

The equations for the turbulent stresses that control the secondary currents for local equilibrium in the presence of vegetation, as well as an open surface yield directly the solutions:

$$
\begin{aligned}
& \overline{v^{2}}=\frac{k}{\left(C_{1}+2 C_{3}\right)}\left\{\frac{2}{3}\left[\left(\alpha-\frac{1}{2} \beta\right)\left(1-\pi_{R}\right)+A_{y y} \pi_{R}+C_{1}-1\right]+\frac{\beta}{\varepsilon}\left(\overline{\nu w} \frac{\partial W}{\partial y}-\overline{u w} \frac{\partial W}{\partial x}\right)\right\}-2 v_{t} \frac{\partial V}{\partial y}, \\
& \overline{u^{2}}=\frac{k}{C_{1}}\left\{\frac{2}{3}\left[\left(\alpha-\frac{1}{2} \beta\right)\left(1-\pi_{R}\right)+A_{x x} \pi_{R}+C_{1}-1\right]+\frac{\beta}{\varepsilon}\left(\overline{u w} \frac{\partial W}{\partial x}-\overline{v w} \frac{\partial W}{\partial y}\right)+C_{3} \frac{\overline{v^{2}}}{k}\right\}-2 v_{t} \frac{\partial V}{\partial x}, \\
& \overline{u v}=\frac{k}{\left(C_{1}+\frac{3}{2} C_{3}\right)}\left\{\frac{\beta}{\varepsilon}\left(\overline{u w} \frac{\partial W}{\partial y}+\overline{v w} \frac{\partial W}{\partial x}\right)+\frac{2}{3} A_{x y} \pi_{R}\right\}-v_{t}\left(\frac{\partial U}{\partial y}+\frac{\partial V}{\partial x}\right)
\end{aligned}
$$


Here, $\pi_{R}$ is the production ratio $\pi_{R}=\pi_{v k} / \varepsilon \quad\left(\varepsilon=\pi+\pi_{v k}\right)$ and $A_{i j}$ is the production anisotropy tensor, here taken as $A_{i j}=0$ for $i \neq j$ and $A_{x x}=A_{y y}=0.88$. More complicated, however, are the equations for the main shear stresses which control the streamwise velocity component $\overline{v w}$ and $\overline{u w}$ :

$$
\begin{aligned}
& \left(C_{1}+\frac{3}{2} C_{3}\right) \overline{v w}=-\frac{k}{\varepsilon}\left\{\left[(1-\alpha) \frac{1}{2}\left(\overline{u^{2}}+\overline{v^{2}}\right)-\beta \overline{w^{2}}+\gamma \quad k\right] \frac{\partial W}{\partial y}+(1-\alpha)\left[\overline{u v} \frac{\partial W}{\partial x}-\frac{1}{2}\left(\overline{u^{2}}-\overline{v^{2}}\right) \frac{\partial W}{\partial y}\right]\right\}+\frac{2}{3} k A_{y z} \pi_{R},(10) \\
& C_{1} \overline{u w}=-\frac{k}{\varepsilon}\left\{\left[(1-\alpha) \frac{1}{2}\left(\overline{u^{2}}+\overline{v^{2}}\right)-\beta \overline{w^{2}}+\gamma \quad k\right] \frac{\partial W}{\partial x}+(1-\alpha)\left[\overline{u v} \frac{\partial W}{\partial y}+\frac{1}{2}\left(\overline{u^{2}}-\overline{v^{2}}\right) \frac{\partial W}{\partial x}\right]\right\}+\frac{2}{3} k A_{x z} \pi_{R}
\end{aligned}
$$

With $A_{y z}=A_{x z}=0$ these may be reformulated as:

$$
\overline{u w}=X_{\mu} v_{t} \frac{\partial W}{\partial x} \quad, \overline{v w}=Y_{\mu} v_{t} \frac{\partial W}{\partial y} \quad \text {, with } \quad v_{t}=C_{\mu} k^{2} / \varepsilon .
$$

For $C_{3}=0$ and $\pi_{R}=0$ it is possible to show that the second brackets of eqs. (10) and (11) become $(1-\alpha)\left(\beta / C_{\mu} C_{1}\right)(\pi / \varepsilon)$ times $\overline{v w}$ and $\overline{u w}$ respectively, turning the choice of $X_{\mu}=Y_{\mu}=1$ possible.

For $C_{3} \neq 0, \pi_{R}=0$, an approximation was suggested [6]:

$$
X_{\mu}=\frac{C_{1}+\frac{5}{2} C_{3}}{C_{1}+2 C_{3}} \quad, \quad \text { and } \quad Y_{\mu}=\frac{C_{1}^{2}}{\left(C_{1}+\frac{3}{2} C_{3}\right)\left(C_{1}+2 C_{3}\right)}
$$

here used adjacent to open surface. With $\pi_{R^{\ddagger}} 0$ the ratio $\pi / \varepsilon$ becomes $1-\pi_{R}$ and an estimation for the common effect of $\pi_{R}$ on the second brackets is obtained. Moreover, note that the first brackets are equal, an etstimation of the effect of $\pi_{R}$ common to both stresses could be evaluated and introduced to the equation for $C_{\mu}:$

$$
C_{\mu}\left(\pi_{R} \neq 0\right)=C_{\mu}\left(\pi_{R}=0\right)\left(\frac{C_{1}+\frac{2}{5} \pi_{R}}{C_{1}}\right)^{2}
$$

\section{HOMOGENEOUS TURBULENCE IN VEGETATED ZONE}

The case of homogeneous, fully developed and steady turbulence produced by vegetation is of particular importance for the tuning of a model for wide vegetated domains. In this limitting case the $k-\varepsilon$ model equations become:

$$
\text { and } \begin{aligned}
& \pi_{v} \eta_{k}-\varepsilon=0 \\
& \pi_{v} \eta_{\varepsilon} C_{\varepsilon 1}-\varepsilon C_{\varepsilon 2}=0 .
\end{aligned}
$$

Since $C_{\varepsilon}$ and $C_{\varepsilon 2}$ were tuned to describe wall dominated flows [3] the two equations can not be satisfied unless:

$$
\eta_{\varepsilon}=\eta_{k} \frac{C_{\varepsilon 2}}{C_{\varepsilon 1}}
$$

Doing so however, turns the $\varepsilon$ equation redundant. To avoid this ambiguity it is suggested to use instead:

$$
\eta_{\varepsilon}=\eta_{k} \frac{C_{\varepsilon 2}}{C_{\varepsilon 1}} \frac{l}{l_{r e f}} \quad, \text { with } \quad l=\frac{C_{\mu^{\frac{3}{4}}}}{\kappa} \frac{k^{\frac{3}{2}}}{\varepsilon}
$$

introducing the $k-\varepsilon$ equations the property of converging solutions with the dissipation length $l$ taking the 
value of $l$ ref. With $\varepsilon$ obtained from the $k$ equation the energy is determined and can be tuned by a proper choice of $\eta_{k}$. Here $\eta_{k}=0.07$ following [8,9], and the choice of $l$ ref will be later discussed.

\section{VEGETATED FLOOD PLAIN}

The mean flow pattern typical of the compound open channel, shown in Fig.2, may change due to the flood plain vegetation. Two cases were elaborated and are shown in Fig.3, for dilute vegetation $<\mathrm{n}>\mathrm{H}^{2}=4$, and in Fig. 4 for more dense vegetation with $\left\langle\mathrm{n}>\mathrm{H}^{2}=25\right.$. In both cases $\langle\mathrm{D}\rangle=0.01 \mathrm{H}$. As expected the streamwise velocity is substautially attenuated almost uniformly all over the flood plain. However, the vortex at the flood plain threshold becomes more intensive, tripling its strength, with its center line being pushed towards the edge of the vegetated flood plain. As a result the location of the streamwise velocity maximum is shiffed away from the flood plain.

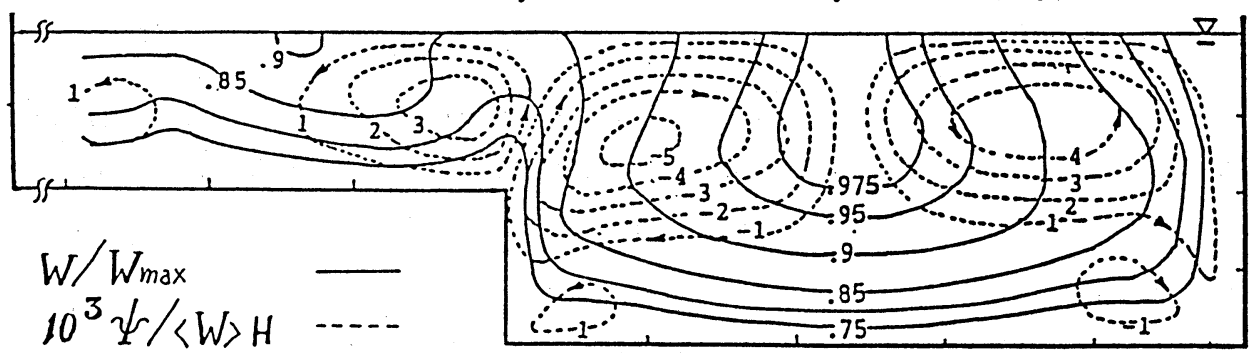

Fig.2. Flow pattern in non vegetated compound open channel

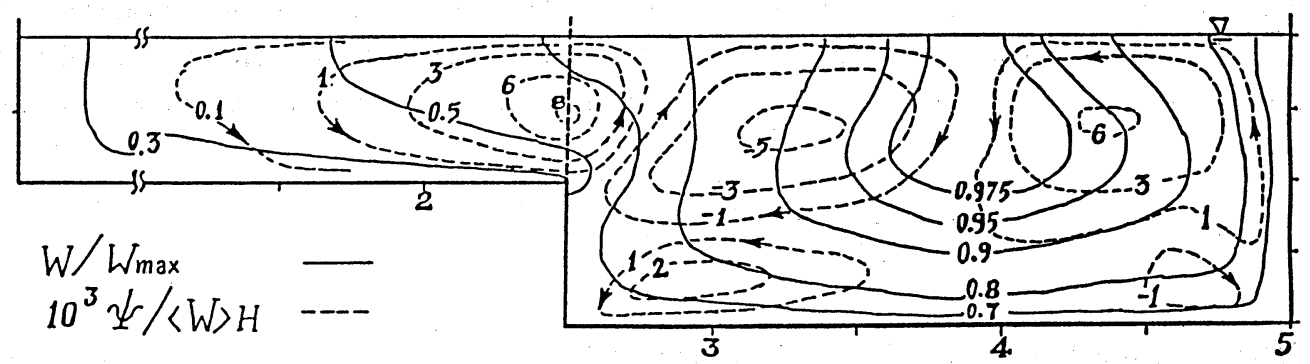

Fig.3. Flow pattern in compound channcl with vegetated flood plain; $<\mathrm{n}>\mathrm{H}^{2}=4$.

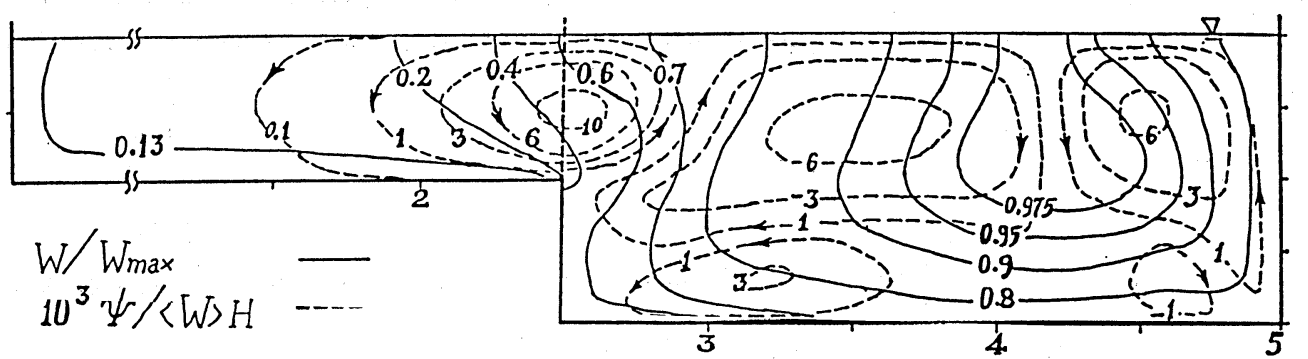

Fig.4. Flow pattern in compound channel with yegetated flood plain; $<n>\mathrm{H}^{2}=25$.

The key to the understanding of the sources of this intensive vortex is the energy distribution which show a large peak, given in Fig.5, at the non vegetated channel close to the flood plain threshold. At this peak the dissipation equation is balanced yeilding dissipation that is $C_{\varepsilon 1} / C_{\varepsilon 2}$ times smaller than the energy production. The energy growth that follows is blocked only by the convection and the diffusion, and is therefore sensitive to the conditions at the adjacent vegetated zone. Two cases are shown in Fig.5, and in Fig.6 for the corresponding dissipation length scale: One, with $l_{\text {ref }}=0.15 H$ typical of the non vegetated similar flood plain, and the second with 
$l_{\text {ref }}=0.01 \mathrm{H}$ which is the averaged diameter of the energy producing vegetation. Limitting the dissipation length at the vegetated flood plain prevents the penetration of the turbulence energy into this zone, and reduce considerably the dimensions of the energy peak. In the absense of an experimental evidence the choice of $l_{\text {ref }}=0.01 \mathrm{H}$ was here practiced, as we expect turbulence to be produced at the turbulent wakes behind the vegetation with scale length typical to these regimes.

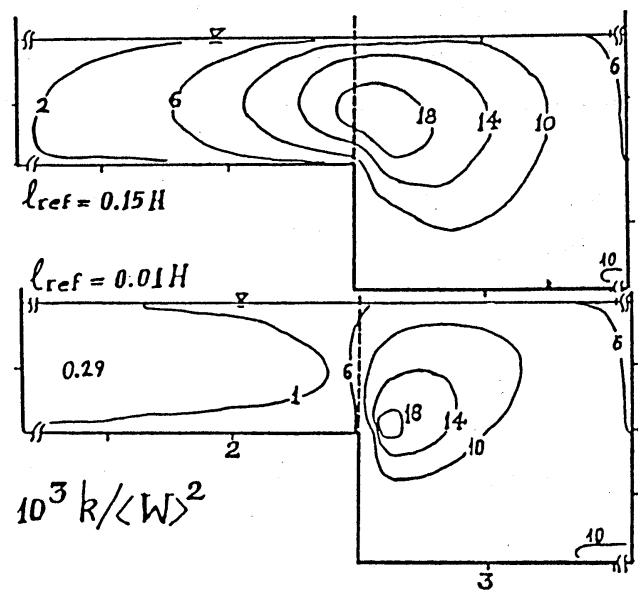

Fig.5. Energy distribution at flood plain threshold.

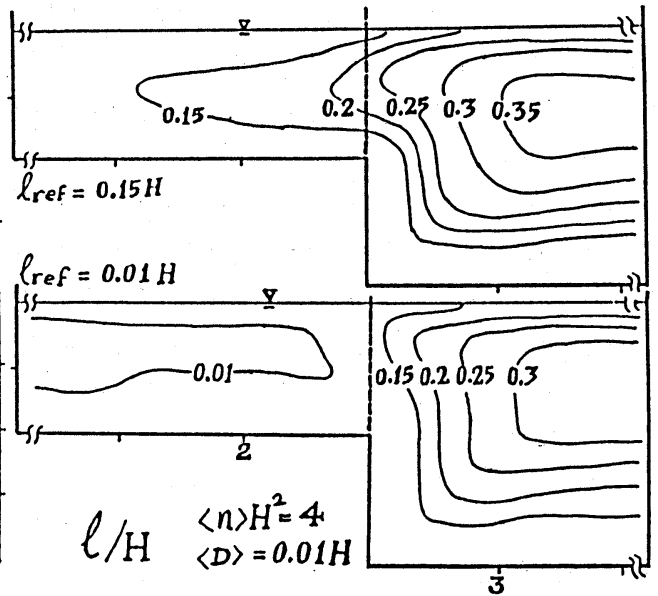

Fig.6. Dissipation length distribution.

The dependence of some mean flow features on the vegetation density is shown in Fig.7. The maximal streamwise velocity at the main channel, and the intensity of the flood plain threshold vortex increase, and the typical streamwise velocity at the flood plain decrease with the increase in the vegetation density. These trends are pronounced for dilute vegetation and are atlenuated for dense vegetation.
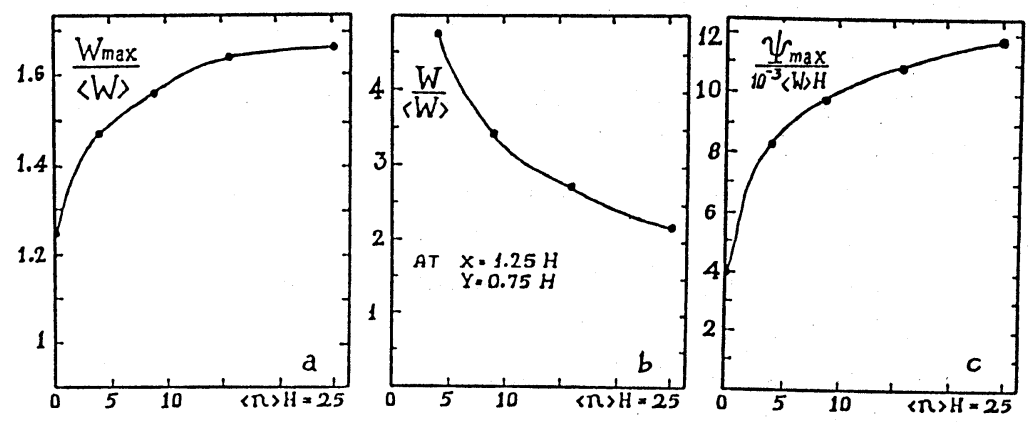

Fig.7. Dependence of: (a) maximum velocity (b) velocity at Flood plain (c) threshold vortex on vegetation density.

\section{CONCLUSIONS}

An intensive longitudinal vortex developes in the non vegetated domain due to the presence of vegetated zone with its center line being close to the edge of the vegetated area. Although the lateral motion is attenuated the secondary currents seem to penetrate considerably deep into the vegetated zone. Pronounced effects on the streamwise velocity distribution due to the influence of this vortex on the secondary currents at the main channel can be observed. 
Both the theoretical discussion and the numerical calculations indicate the extreme importance of the choice of the efficiencies of the production of turbulence energy and dissipation, in determinig the structure of turbulence and the flow pattern at the vegetated flood plain as well as at the non vegetated main channel. Comparison with experimental data in simple basic cases is highly recommended.

The tuning of the $k-\varepsilon$ model with the energy equation being at local equilibrium and the dissipation equation being under balanced leaning by diffusion on the wall boundary conditions is not applicable at a large vegetated domain. Doubts to whether this is also the case at the flood plain threshold were here raised calling for confirmation on base of experimental data. Recent attempts to isolate a free shear layer close to the edge of a vegetated layer produce simmilar impression.

Although a proposal was made to distinguish between the resistance to motion paralel to the vegetation direction and the resistance to the flow in perpendicular, the option was almost idle as the vegetation Reynolds number was relatively small. Further study of this issue is needed.

Former experience $[4,5]$ showed that the longitudinal vortices are sensitive to the ratio between $\mathrm{dx}$ to $\mathrm{dy}$, suggesting that this ratio be limitted to values smaller than 1.25 which correspond to 5 percents error. It also showed that further refinement in the $24 \times 120$ grid may intoduce errors due to the use of a high Reynolds number wall functions at a too low $y+$ which may over rule the accuracy gained by the refinement.

\section{Acknowledgement}

The research was performed at the Dept. of Civil Engrg., Div. of Global Environment Engrg., Graduat School of Engrg., Kyoto University, Kyoto, Japan, and was financially supported by the Asahi Glass Foundation. The stay of the first author was supported by the Kajima Foundation. The financial support is hereby acknowledged.

\section{REFERENCES}

1. Kawahara, Y., and Tamai, N. (1989), "Proposal of a New Algebraic Stress Modeling of Flood Plain Flows", J. Hydr. Engrg., ASCE, 112(4), 251-267.

2. Krishnappan, B. G., and Lau, Y. L. (1986), "Turburence Modelling of Flood Plain Flows", J. Hydr. Engrg., ASCE, 112(4), 251-267.

3. Launder, B.E., and Spalding, D.B. (1974). "The Numerical Comptation of Turbulent Flow", Comp. Method in Appl. Mech. and Engrg., 3, 269-289.

4. Naot, D., Nezu, I., and Nakagawa, H. (1993), "Hydrodynamic Behaviour of Compound Rectangular Open channels", J. Hydr. Engrg., ASCE, 119(3), 390-408.

5. Naot, D., Nezu, I., and Nakagawa, H. (1993), "Calculation of Compound Open Channel Flow",J. Hydr. Engrg., ASCE, 119(12), 1418-1426.

6. Naot, D., and Rodi, W. (1982), "Calculation of Secondary Currents in Channel Flow", J.Hydr.Div., ASCE, 108(8), 948-968.

7. Prinos, P., Townsend, R., and Tovoularis, S. (1985), "Structure of Turburence in Compound Channel Flow", J. Hydr. Engrg., ASCE., 111(9), 1246-1261.

8. Shimizu, Y., Tsujimoto, T., and Nakagawa, H. (1992), "Numerical Study on Fully Developed Turbulent Flow in Vegetated and Non Vegetated Zones in a Cross Section of Open Channel", Proc. of Hydr. Engrg., JSCE, 36, 265-272.

9. Shimizu, Y., Tsujimoto, T. (1993), "Comparison of Flood Flow Structure Between Compound Channel and Channel With Vegetated Zone", Proc. 25th. congress of IAHR, Tokyo, Japan, A-3-4, 97-104. 